\title{
An Analysis Framework for Mobile Workforce Automation
}

\author{
Volker Gruhn \\ University of Leipzig \\ Department of Computer Science \\ Chair of Applied Telematics / e-Business \\ Klostergasse 3, 04109 Leipzig, Germany \\ gruhn@ebus.informatik.uni-leipzig.de
}

\author{
André Köhler \\ University of Leipzig \\ Department of Computer Science \\ Chair of Applied Telematics / e-Business \\ Klostergasse 3, 04109 Leipzig, Germany \\ koehler@ebus.informatik.uni-leipzig.de
}

\begin{abstract}
In this paper we introduce an analysis framework for mobile workforce automation. The framework is based on the findings from earlier research as well as on an analysis of 27 recent case studies conducted within the field of mobile workforce automation. It consists of a general reference process for mobile work and of a model explaining influencing factors (worker, task, coordination system, information system), optimization goals and their relationships in mobile business processes. The framework can be applied to process modeling, simulation, and optimization as well as to requirements analysis and return on investment calculations. Based on the results of case study evaluation, it is furthermore shown, that recent mobile IT solutions are mainly built for relatively simple processes and cooperation models. Mobilizing more complex processes still seems to be a challenge.
\end{abstract}

\section{Introduction}

Mobile B2E-applications (business-to-employee) can add significant value to a company's business, when large workforce divisions are involved into the enactment of business processes. Altough, from a technical point of view, the major issues are solved (e.g. solutions for connectivity, broadband mobile networks, synchronization mechanisms, secure protocols etc.). From a business point of view, there is a lack of methodology regarding the alignment of technical solutions to business needs. As companies face a continuously faster change concerning business models, legal constraints, and customer needs, highly flexible systems are needed to react to changing business processes. Furthermore, the introduction of new mobile systems is often a technology-driven process, pushing the alignment of software and systems to the highly specific, fast changing business needs into the background.
The aim of this article is to show that due to several characteristics caused by the workers mobility, a general industryindependent mobile process can be established allowing to describe most of today's mobile processes. Furthermore, the most frequent characteristics of mobile work are described, which can be used to refine the general process in order to model an industry- or company-specific process. The findings are summarized in a framework, which can be applied to process modeling, simulation, and optimization. Additionally, they can serve as a basis for requirements analysis and return on investment calculations as well. The framework is based on a literature review of 27 recent case studies published in scientific journals and conference proceedings.

This paper is organized as follows: Section 2 gives an overview about the related work. Section 3 describes the developed framework explaining the reference work process (3.1), influencing factors on the process $(3.2,3.3)$, and general optimization goals (3.4). Section 4 shows the results of the case study evaluation. Possible framework usage scenarios are described in section 5. Section 6 draws a conclusion and gives an outlook on further research.

\section{Related Work}

In [18] some of the changes within the discipline of software engineering, concerning the development of systems for mobile environments, are discussed. The authors state that "mobility represents a total meltdown of all stability assumptions [...] associated with distributed computing". A comprehensive overview of software engineering for mobile systems is given regarding issues like models, algorithms, applications and middleware.

A conceptual analysis of the term mobility is provided by [8]. Within the context of mobile applications the term is typically used meaning spatial mobility. Kakihara and Sorensen expand this concept to temporal and contextual mobility. Amongst others, temporal mobility describes the 
fact that "it is no longer strictly necessary to share the same time period exclusively with a particular person or group" [8]. To put it differently, the term describes the absence of temporal constraints through the use of mobile technologies. Furthermore, contextual mobility is given when contextual constraints for a certain task of a mobile worker can be avoided, e.g., when he carries the necessary information to the place of task completion. This work gives an excellent analytical basis for further work on the mobility concept.

The concept of mobility is also subject of [21] in which user mobility is distinguished into personal and terminal mobility. The paper gives an overview about recent developments at OSI network levels for improving terminal mobility, i.e., connectivity. Moreover, the authors present an integrated personal mobility architecture which supports personal mobility. This architecture aims at context preservation during device or network changes. On the basis of these key factors, concrete implications for the general design of mobile systems are given. The particular importance of context is also subject of [6]. Different types of context in mobile environments like the infrastructure context, the system context, the domain context, and the physical context are discussed. On this basis, a design framework for mobile systems is presented, which can be used to develop models of space and awareness and to deduce requirements for software architectures. [15] also presents results regarding the basic concept of mobility. Within this study different aspects of mobile work in general are examined. The four key factors identified for mobile work are the role of planning, working in dead time, accessing remote resources, and monitoring distant activities.

A number of recent case studies show that efficiency and effectiveness of business processes can be improved by using mobile technologies (see Table 2 and discussion in 5). [23] state that benefits of mobile technology are difficult to quantify. Further on the authors deduce several propositions from their case studies. The first proposition is that benefit is gained when coordination is required for actors who are difficult to locate. The second proposition is that the benefit of a mobile solution is to avoid opportunity costs which occur due to not being able to coordinate actors. The presented methodology for analyzing mobile processes in [4] requires the four steps identify ten processes, map out the as-is-process, map out the to-be-process, and select four processes. The method is evaluated using site engineer processes. A business process analysis for the electricity industry is presented in [10]. Here the authors introduce a method for identifying and modeling selected business processes for a specific goal. The method consists of a knowledge model, a goal submodel, and a process submodel containing actors, roles, activities, and resources. Based on these tools, concrete goals and process models for a company can be cre- ated followed by an analysis of these results. The authors of [16] recommend to strongly focus on the type of mobility when dealing with mobile workers in order to design the right mode of interaction. Further, it is proposed to make a distinction between structured and unstructured work when designing mobile systems.

[26] provide an analysis framework focusing on assessing the design approach of mobile workforce solutions. Within this analysis framework, the four perspectives thinking, controlling, working, and modeling are distinguished. [12] presents an analysis of requirements for mobile systems within the construction industry. Starting from defined situations, the information needed on site, the information created on site, and the involved people are identified. Furthermore, core business functions for specific problem situations are developed. In [19] a model for assessing the readiness prior to IS investment is described. [14] presents results of a case study conducted with a utility company. On the basis of interviews with the company's employees, the authors developed a means-ends objective network that can be used to increase the value of a mobile application.

\section{An Analysis Framework for Mobile Work- force Automation}

As described in section 2, a number of papers and case studies report on successful implementations of mobile systems in different business contexts. Based on this research as well as on the authors' experiences from similar projects, a couple of repeatedly recurring process and system characteristics can be observed. In the following, these characteristics are described and a reference model for mobile business processes is designed. This model can be used for different purposes analyzing and designing mobile business processes and systems (see section 5). Amongst others, such goals could be process analysis for optimization potentials, requirements analysis for mobile systems, or return on investment analysis for mobile systems.

\subsection{General Mobile Work Process}

As stated above, within a wide range of case studies (see Table 2) a typical pattern for mobile work processes becomes apparent. This pattern is applicable in different companies and industries. This general mobile work process is shown in Figure 1. The process is based on four major components:

- A company, which requires the execution of defined mobile tasks.

- A group of mobile workers (mobile workforce). 


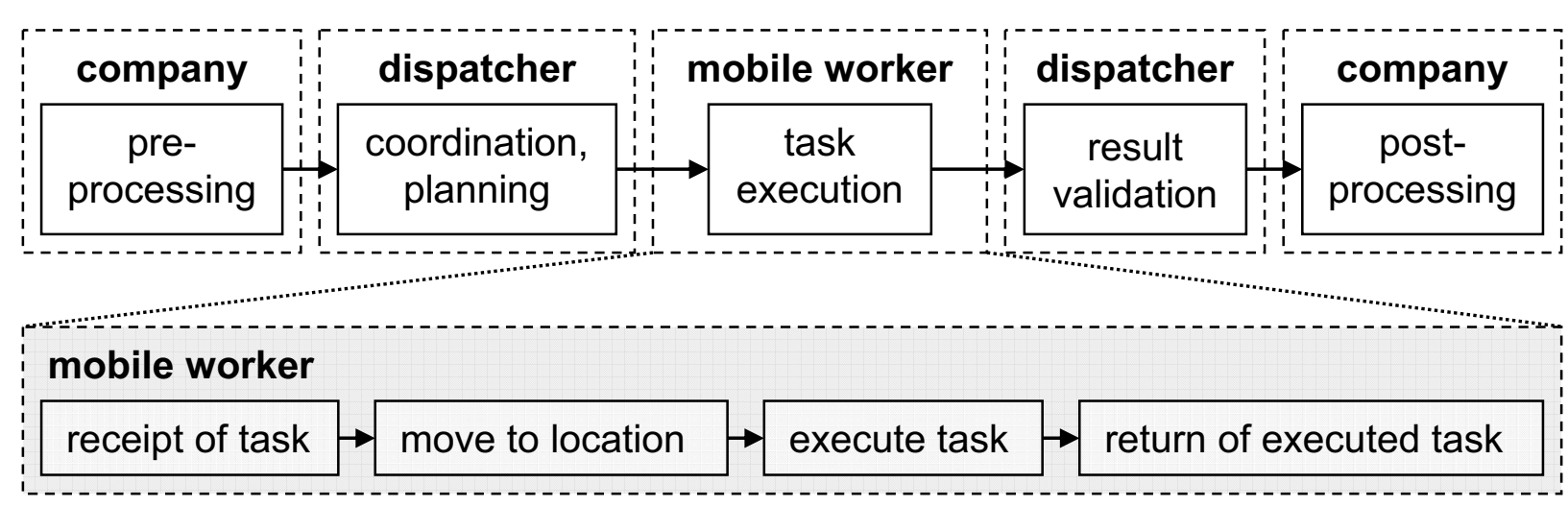

Figure 1. General mobile process.

- A dispatcher, which coordinates mobile workers and tasks.

- A task-centered work process of the mobile worker.

The process is structured as follows: The company creates tasks and sends them to a dispatcher that coordinates its mobile workers. The dispatcher receives tasks from the company and sends each to an appropriate worker. The worker receives it, moves to the specified location, executes the task and returns the task result back to the dispatcher. The dispatcher receives the task result and sends it to the company, where subsequent processes can be initiated.

As a result from analyzing the related work, case studies and reports, this model can be applied for the vast majority of mobile work processes. The dispatcher and the mobile workforce are not necessarily required to be part of the company, but could be external service providers as well. However, the above described process is a very simplified process, prescinding from any details or variant. Yet, the nature of mobility bears opportunities as well as constraints when modeling a more detailed process. Analyzing the constraints the authors identified typical factors influencing the process design. They can be distinguished into factors caused by the organizational structure and the business model, and into factors caused by the use of information technology. Furthermore, a couple of general optimization goals could be extracted when redesigning such processes or implementing mobile systems. The influencing factors as well as the optimization goals are shown in Figure 2 and will be described in the following.

\subsection{Influencing Factors based on Or- ganizational Structure and Business Model}

The main influencing factors on mobile business processes arising due to the organizational structure and the business model of the company are worker and task. Both influencing factors as well as their effects for the above described process are explained as follows.

\subsubsection{Worker}

The first distinction can be made assuming that the company's mobile workforce is not a volume of unique subjects but workers with different skills. Correspondingly, a task could require specific skills from the executing worker. Furthermore, a mobile worker could be associated with a certain geographical area, that he can not leave or just at higher costs. In addition, the company's mobile workforce could be temporarily extended by subcontractors, which might e.g. result in higher costs for task completion.

The autonomy of the mobile worker can be considered if the worker is allowed to reject a task assigned by the dispatcher. In this case, the dispatcher needs to regard this fact during task planning. This would be especially important if subcontractors participate. Related to the autonomy aspect, the types of task handling can be divided into sequential and parallel ones. Sequential task handling means to be required to close the current task before the next can be started, even if the task has not been completed. In this case, the dispatcher could later assign this task to the same worker or to another. Parallel task handling allows the worker to choose from a couple of tasks to complete and in particular to restart the processing of a task at a later time.

\subsubsection{Task}

The majority of mobile tasks might be of simple nature and can be completed by a single worker in a short time. But some tasks may require to cooperate with several specialists. Two major kinds of cooperation are particularly important: sequential cooperation (multiple mobile workers execute the task in a defined order) and team cooperation 


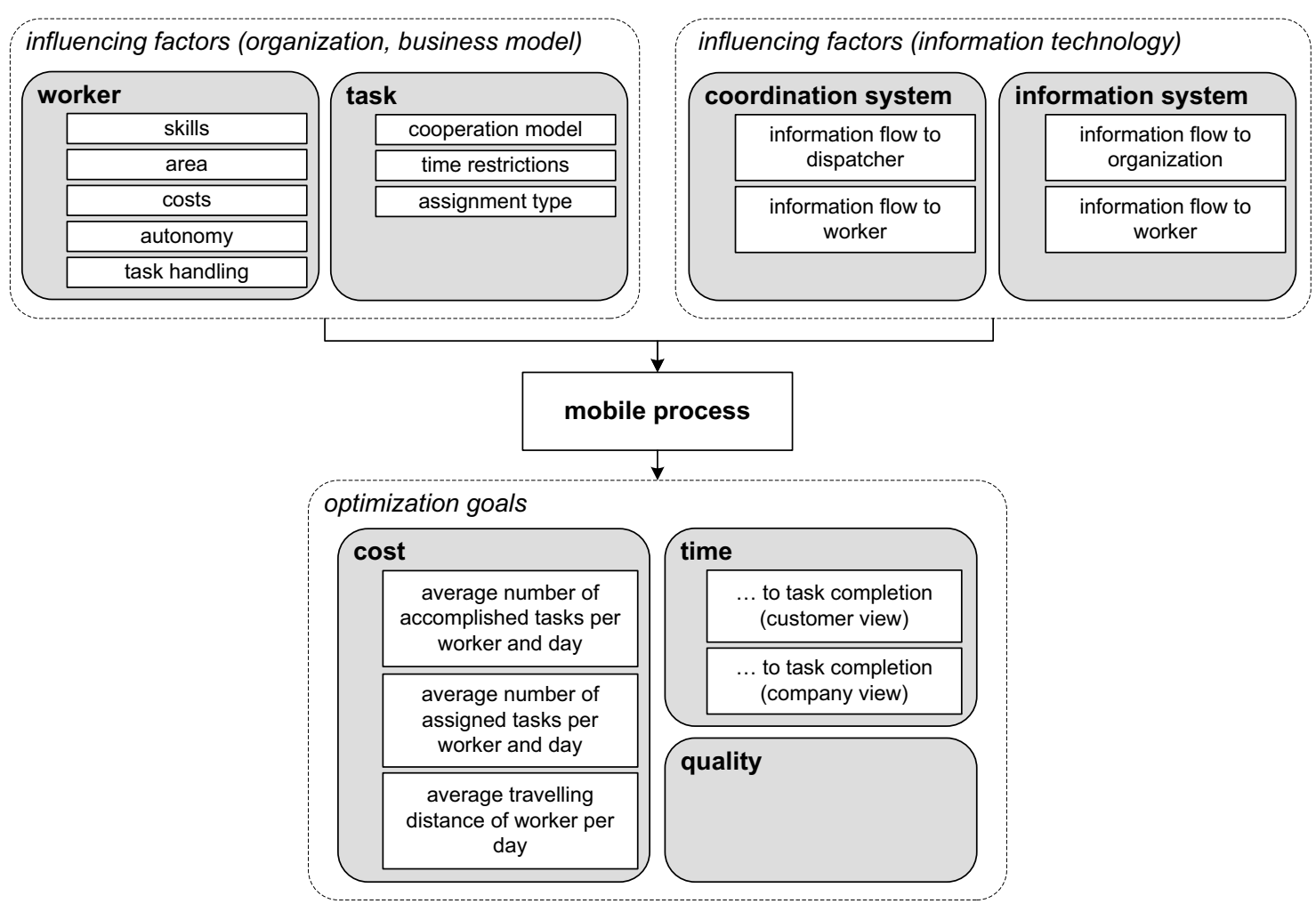

Figure 2. Influencing factors and optimization goals.

(multiple mobile workers execute the same task at the same time or ad hoc in undefined order).

The highest degree of flexibility for planning and executing a task is given when no time restriction exists. But often a task is associated with a deadline until it has to be completed. Even more restrictive is a task if it has a set execution date (e.g., appointments). Furthermore, it can be necessary to fulfill a task immediately.

One way of task allocation is assigning a single task to a single worker. A different way is to assign a couple of tasks (e.g., for a day) to a single worker. The type of assignment is one influencing factor on the frequency of communication between worker and dispatcher.

\subsection{Influencing Factors due to Mobile In- formation Technology}

The type of support for mobile workers by information technology can mainly be distinguished into coordination systems and information systems. A coordination system is used to build schedules and plan routes with regard to certain optimization goals. Furthermore, the system is often used to submit tasks and to control worker and task state. An information system is needed in order to connect the mobile worker with the company's systems and to supply the worker with necessary data and functionalities at the point of action. The information system is used to retrieve, manipulate and store necessary information. As a couple of case studies report, mobile systems often realize both functionality in one physical system. However, the separation of both aspects is helpful when analyzing processes and designing systems.

\subsubsection{Coordination System}

Using a coordination system, several options for the process design arise. One is to realize the information flow to the dispatcher. The coordination system could therefore constantly transmit information to the dispatcher concerning the actual geographical location of the worker as well as the actual state of the worker or the task. This information could be used for ad hoc planning and task assignment. Furthermore, the information flow to the worker can be supported. This is often the main purpose of the coordination system because it delivers tasks to the mobile worker.

The coordination system consists of two major parts. The dispatcher is responsible for

- assigning tasks to workers,

- planning routes, 


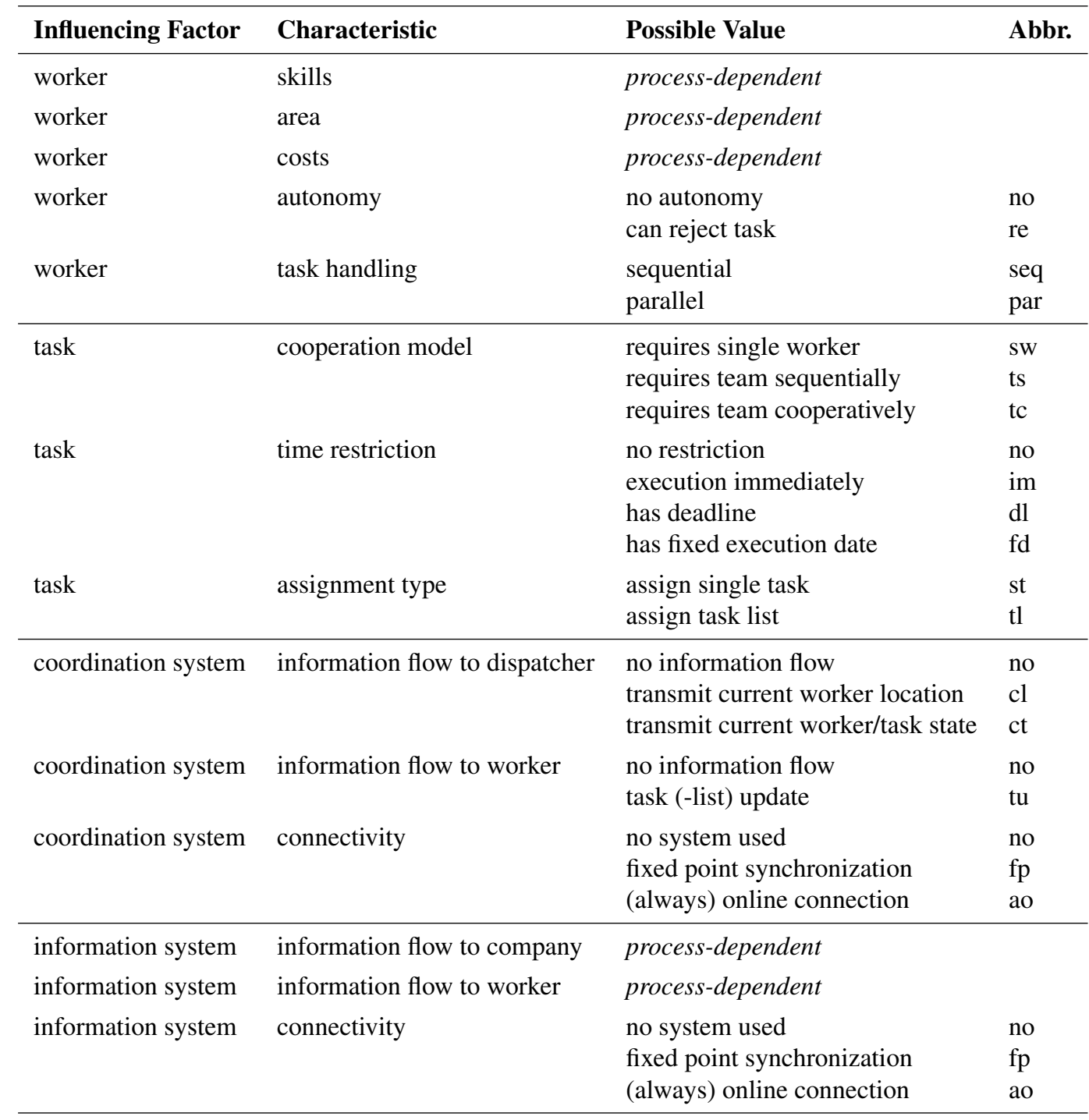

Table 1. Influencing factors on process and system design.

- controlling workers load and state,

- controlling task fulfillment,

- receiving task result from worker

and many more. The workers-layer must support

- receiving new tasks,

- managing current tasks and

- sending task result to dispatcher.

An extended function of the system could be, e.g., to send the GPS coordinates of the worker's current location to the dispatcher permanently for real time planning purposes.
Furthermore, coordination systems can be distinguished by their connectivity into (always) online systems, connecting workers nearly constantly with the dispatcher via a mobile network, and offline systems, where workers have to move to fixed points (e.g., home office) in order to synchronize their applications with a central server at the dispatcher site.

\subsubsection{Information System}

An information system can be used to provide access to data and applications to mobile workers on the road. It focusses on supporting the fulfillment of tasks by workers. The first major function is to support the information flow to the worker. The higher the complexity of a task, the higher 


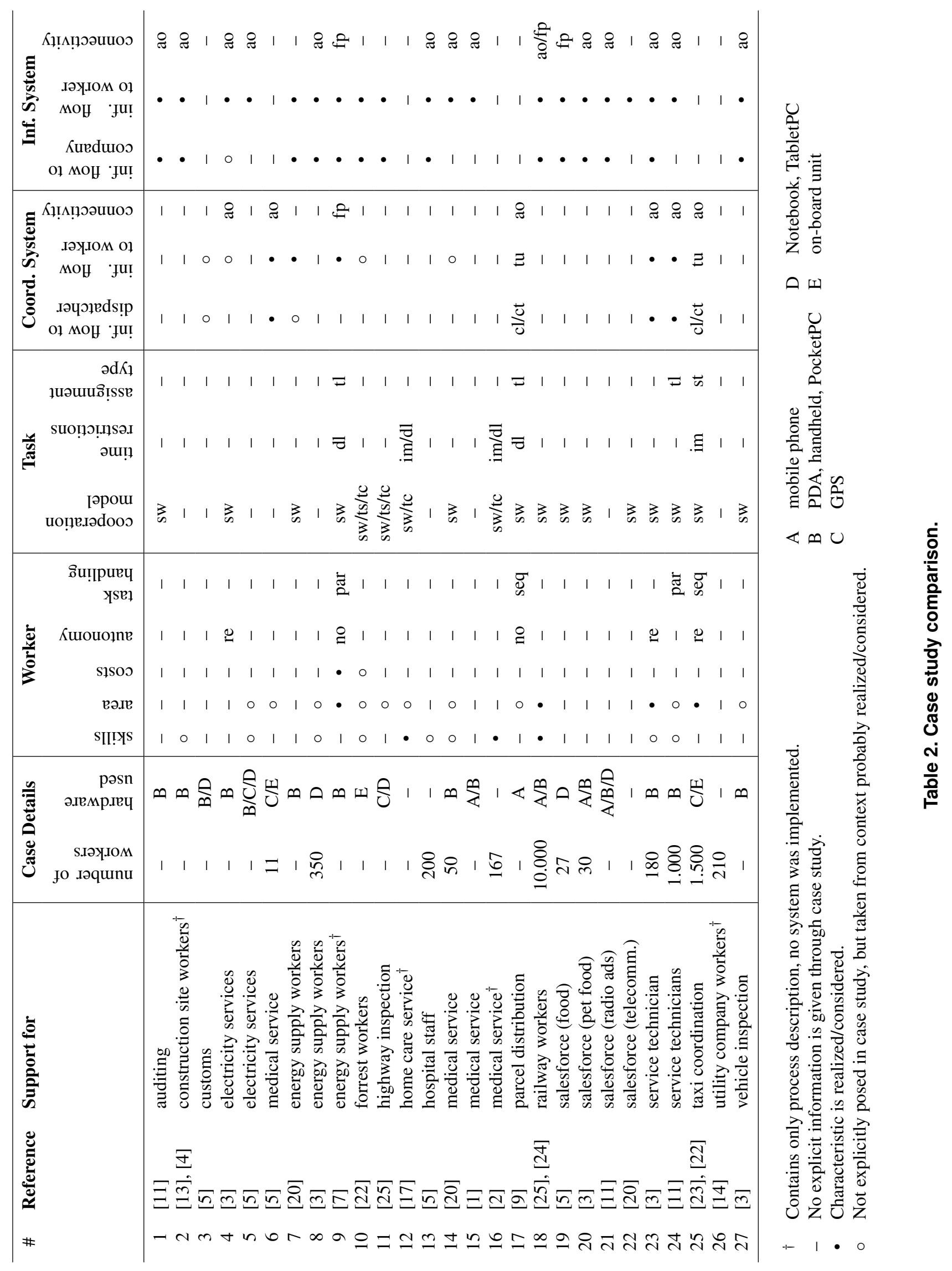


is the likelihood of needing unpredictable information at the point of action. Information systems can be used to provide such information to the mobile worker whenever the worker needs it, avoiding additional trips to fetch the necessary information. The second major function is to support the information flow to the organization. If a task requires the acquisition of information, the system can automatize the electronic gathering and (online) submission to the dispatcher. This allows for faster processing of acquired data. Formerly necessary media conversions are avoided, plausibility checks become possible.

As described for coordination systems, information systems can be separated by their connectivity into (always) online systems in which workers are connected nearly constantly with the company via a mobile network, and offline systems in which workers have to move to fixed points (e.g. home office) in order to synchronize their applications with a central server at the company.

\subsection{Optimization goals}

As above stated, mobile processes are task-based and principal/agent-oriented. Different process variants occur due to different influence factors. When introducing or changing mobile systems (for coordination and/or information purposes) within such a process, the company aims at achieving certain optimization goals. An optimization goal always belongs to one of the categories cost, time or quality referring to the process outcome.

Typical cost optimization goals are to increase the average number of accomplished tasks per worker and day, to increase the number of assigned tasks per worker and day and to decrease the average traveling distance of worker per day. Typical time optimization goals are to minimize the average time until task completion, either from the point of the customer or the company. For both categories more goals are conceivable.

Whereas these are quantitative measures, the quality category usually contains company- and process-specific measures regarding the process outcome. These could be, e.g., the avoidance of preparing and concluding processes, the avoidance of recurrent visits, and the ability to immediately and fully deliver the requested information to the point of action.

\section{Case Study Evaluation}

\subsection{Evaluation Approach}

The different influencing factors and their possible values described above are summarized in Table 1. Together with the structure presented in Figure 2 and the process descrip- tion illustrated in Figure 1 it constitutes the analysis framework for mobile workforce solutions.

The design of this framework is mainly influenced by reportings from case studies concerned with the design and implementation of IT solutions for mobile processes. Table 2 shows the evaluation results of 27 recent case studies, mapped to the above explained framework. For reasons of trust and quality, we considered only reportings that appeared in scientific journals and conference proceedings since 2000. The large amount of white papers and project reports produced by IT companies specialized on selling mobile solutions are explicitly not considered.

Table 2 shows some general description for each case study. Furthermore, it indicates whether or not the case study reports about the consideration of the above described influencing factors. If a case study reports on the realization of a framework aspect, this is indicated by ' $\bullet$ '. If the realization of a framework aspect is not explicitly posed in a case study, but taken from context probably realized or considered, this is indicated by 'o'. The icon '-' shows that no explicit information is given by the case study. If the realization of a framework aspect is described in more detail, the abbreviations given in the most right column of Table 1 are used.

\subsection{Evaluation Results}

Comparing the different case studies, we came to the following findings. In some industries the adoption of mobile solutions is notably higher than in others, namely, for electricity services and energy supply workers, for medical services as well as for salesforces. Furthermore, it can be observed that the number of workers affected by a mobile solution ranges from $10-10.000$. The used hardware is often a PDA, handheld or PocketPC, whereas the use of notebooks or Tablet PCs for mobile workforces seems to be rather unusual. The consideration of workers skills and current location is described explicitly only in a few case studies. Only one case study reports on the option of different costs for different workers. Regarding the autonomy of mobile workers, only three case studies consider of worklers being able to reject dispatched tasks. Only a few case studies mention the option of task handling. Nearly all case studies report about a "single-worker" cooperation model, in which actually no cooperation occurs. Only four cover teamwork. Regarding time restrictions and the assignment type, only few data is given. About the design of the coordination system, case studies often do not give explicit information. Information flow to the mobile worker is often stronger supported than the information flow to the dispatcher. The connectivity of the coordination system is often realized through an always-online connection. In contrast, a lot of case studies report on using information sys- 
tems. The information flow to the mobile worker as well as to the company is realized well. The connectivity of the information system is often also established through an always-online connection.

Given these results, some conclusions can be drawn. Firstly, it becomes obvious that most case studies give just a samll amount of information needed for case evaluation on the basis of the presented framework. It remains unclear whether certain aspects were not considered in a project or if the authors simply did not report on it. Hence, future case studies in this area of research could find the introduced framework helpful when structuring reports. Secondly, evaluation results show that mobile solutions are mainly built for simple processes and tasks with low complexity concerning coordination and information supporting aspects. In almost all case studies, the workforce is homogenus. Nearly no case study takes into consideration different costs per worker or decision autonomy (task rejection etc.). Besides a few exceptions, the cooperation model is mainly based on the single-worker-approach, nearly no processes with team cooperation can be observed. This could lead to the assumption that it is a general mobile processes characteristic. That might be the case for the majority of processes, but we believe that mobile IT solutions for complex mobile business processes with underlying team cooperation models and difficult coordination aspects are just arising. Thirdly, it becomes obvious that today's mobile IT solutions focus on information support of mobile workers. Relatively little seems to be done regarding the use of coordination systems in order to improve the efficient employment of mobile workers.

\section{Framework Usage}

The model illustrated in Figure 2 shows the essentials for understanding the interdependencies between mobile business processes and mobile technologies. Center of the model is the mobile process, producing an outcome with value for the company. The constraints determining the process outcome are shown by the organizational structure and the underlying business model as influencing factors (see the box in the upper left corner). The process outcome is produced under these constraints and is characterized by management ratios or optimization goals (see the box at bottom). This means that the company constantly produces the process outcome under the given constraints but aims at improving the optimization goals. The variable part to achieve this objective is the kind of support with information technology (changes in organizational belongings or business development could also lead to this goal, but are not in scope of this paper). Thus, two major conclusions can be drawn. Firstly, when supporting information technology is about to be improved or to be introduced into the process, resulting changes concerning optimization goals have to be pointed out clearly. Secondly, the applied information technology needs to be effectively adjusted to the given organizational and business constraints. Aspects of both conclusions are elaborated in the following paragraphs.

With a detailed modeling of the mobile process, several goals can be achieved. Analyzing the requirements of mobile process supporting information technology, the introduced framework can help to show most typical constraints due to organizational structures and business models. Furthermore, it helps to distinguish between information and coordination aspects, showing typical functions to realize for mobile environments. The modeled process can further be used for, e.g., simulation purposes in order to identify whether changes to technology have desired effects. Parameters of the simulation are given through the above explained reference model. Thus, a clear frame for process modeling, simulation, and optimization purposes is given.

\section{Conclusion and Outlook}

In this paper we presented a framework for analysis purposes within the domain of mobile business processes and related systems. The aim of the framework is to summarize the findings from previous work related to research and case studies regarding mobility. The framework can be used for different purposes in conjunction with B2E-processes and applications, e.g., supporting requirements analysis as well as the modeling, simulation, and optimization of processes. It consists of a general mobile work process as well as of a model describing the relationship between the work process, influencing factors, and optimization goals.

Further research is necessary to increase efficiency and impact of mobile information technology on mobile business processes. From a technical point of view, the major issues are solved (e.g. solutions for connectivity, broadband mobile networks, synchronization mechanisms, secure protocols etc.). Yet, from a business point of view, there is a lack of methodology regarding the alignment of technical solutions to business needs. As companies face a continuously faster change in business models, legal constraints, and customer needs, highly flexible systems are required that are able to react to changing business processes. The authors see a great demand for process simulation, considering current and future changes in organizational, business, and technological constraints. This is an optimization problem with multiple optimization goals and a large variety of input parameters. Further research on this topic is planned.

\section{Acknowledgments}

The Chair of Applied Telematics/e-Business is endowed by Deutsche Telekom AG. We would like to thank Sören Blom 
for his valuable comments.

\section{References}

[1] E. Ammenwerth, A. Buchauer, B. Bludau, and R. Haux. Mobile information and communication tools in the hospital. International Journal of Medical Informatics, 57(1):2140, 2000.

[2] J. E. Bardram and C. Bossen. Moving to get ahead: Local mobility and collaborative work. In Proceedings of the Fifth European Conference on Computer Supported Cooperative Work (ECSCW2003), pages 355-374, 2003.

[3] S. J. Barnes. Wireless support for mobile distributed work: A taxonomy and examples. In Proceedings of the Proceedings of the 37th Annual Hawaii International Conference on System Sciences (HICSS'04) - Track 3 - Volume 3. IEEE Computer Society, 2004.

[4] S. Bowden, A. Dorr, A. Thorpe, and C. J. Anumba. Mapping site processes for the introduction of mobile IT. eWork and eBusiness in Architecture, Engineering and Construction. Taylor and Francis Group, London, 2004.

[5] T. Brodt and R. Verburg. Managing mobile work - insights from european practice. New Technology, Work and Employment, 2007. University of St.Gallen, Blackwell Publishing.

[6] A. Dix, T. Rodden, N. Davies, J. Trevor, A. Friday, and K. Palfreyman. Exploiting space and location as a design framework for interactive mobile systems. ACM Transactions on Computer-Human Interaction, 7(3):285-321, 2000.

[7] V. Gruhn, A. Köhler, and R. Klawes. Modeling and analysis of mobile service processes by example of the housing industry. In W. M. v. d. Aalst, B. Benatallah, F. Casati, and F. Curbera, editors, Proceedings of the International Conference on Business Process Management (BPM 2005), LNCS 3649, Nancy, France, 2005. Springer.

[8] M. Kakihara and C. Sorensen. Expanding the 'mobility' concept. SIGGROUP Bulletin, 22(3):33-37, 2001.

[9] M. Kakihara and C. Sorensen. Mobility: An extended perspective. In Proceedings of the 35th Annual Hawaii International Conference on Systems Sciences (HICSS-35'02), pages 1756-1766, 2002.

[10] E. V. Kavakli and P. Loucopoulos. Goal-Driven Business Process Analysis - Application in Electricity Deregulation. In CAiSE '98: Proceedings of the 10th International Conference on Advanced Information Systems Engineering, pages 305-324, London, UK, 1998. Springer.

[11] A. Kornak, J. Teutloff, and M. Welin-Berger. Enterprise Guide to Gaining Business Value from Mobile Technologies. John Wiley and Sons, 2004.

[12] A. May, V. Mitchell, S. Bowden, and T. Thorpe. Opportunities and challenges for location aware computing in the construction industry. In MobileHCI '05: Proceedings of the 7th international conference on Human computer interaction with mobile devices \& services, pages 255-258, New York, NY, USA, 2005. ACM Press.

[13] A. May, V. Mitchell, S. Bowden, and T. Thorpe. Opportunities and challenges for location aware computing in the construction industry. In Proceedings of the 7th international conference on Human computer interaction with mobile devices and services, pages 255-258, Salzburg, Austria, 2005. ACM Press.

[14] F. F.-H. Nah, K. Siau, and H. Sheng. The value of mobile applications: a utility company study. Communications of the ACM, 48(2):85-90, 2005.

[15] M. Perry, K. O'hara, A. Sellen, B. Brown, and R. Harper. Dealing with mobility: understanding access anytime, anywhere. ACM Transactions on Computer-Human Interaction, 8(4):323-347, 2001.

[16] D. Pica, C. Sorensen, and D. Allen. On Mobility and Context of Work: Exploring Mobile Police Work. In HICSS '04: Proceedings of the 37th Annual Hawaii International Conference on System Sciences (HICSS'04) - Track 3, Washington, DC, USA, 2004. IEEE Computer Society.

[17] D. Pinelle and C. Gutwin. Designing for loose coupling in mobile groups. In Proceedings of the 2003 international ACM SIGGROUP conference on Supporting group work, pages 75-84, Sanibel Island, Florida, USA, 2003. ACM Press.

[18] G.-C. Roman, G. P. Picco, and A. L. Murphy. Software engineering for mobility: a roadmap. In ICSE '00: Proceedings of the Conference on The Future of Software Engineering, pages 241-258, New York, NY, USA, 2000. ACM Press.

[19] Y. Saleh and M. Alshawi. An alternative model for measuring the success of IS projects: the GPIS model. Journal of Enterprise Information Management, 18(1):47-63, 2005.

[20] R. Schierholz, L. Kolbe, and W. Brenner. Strategy alignment of mobile solutions in customer-oriented processes. Wirtschaftsinformatik, 47(1):17-24, 2005.

[21] B. Thai, R. Wan, A. Seneviratne, and T. Rakotoarivelo. Integrated personal mobility architecture: a complete personal mobility solution. Mobile Networks and Applications, 8(1):27-36, 2003.

[22] P. Valiente and H. van der Heijden. A method to identify opportunities for mobile business processes. Technical Report 2002:10, Stockholm School of Economics, 2002.

[23] H. van der Heijden and P. Valiente. The value of mobility for business process performance: Evidence from sweden and the netherlands. In European Conference on Information Systems (ECIS), 2002.

[24] Y. Wang, E. v. d. Kar, and G. Meijer. Designing mobile solutions for mobile workers: lessons learned from a case study. In Proceedings of the 7th international conference on Electronic commerce, pages 582-589, Xi'an, China, 2005. ACM Press.

[25] Y. Wang, E. v. d. Kar, G. Meijer, and M. Hunteler. Improving business processes with mobile workforce solutions. In Proceedings of the International Conference on Mobile Business (ICMB'05), pages 94-102. IEEE Computer Society, 2005.

[26] Y. Wang, E. van de Kar, and G. Meijer. Designing mobile solutions for mobile workers: lessons learned from a case study. In ICEC '05: Proceedings of the 7th international conference on Electronic commerce, pages 582-589, New York, NY, USA, 2005. ACM Press. 